\title{
Genome sequence of a commensal bacterium, Enterococcus faecalis CBA7120, isolated from a Korean fecal sample
}

\author{
Joon Yong Kim ${ }^{1}$, Hye Seon Song ${ }^{1,2}$, Yeon Bee Kim ${ }^{1,2}$, Joseph Kwon' ${ }^{1}$, Jong-Soon Choi ${ }^{1}$, Yong-Joon Cho ${ }^{3}$, \\ Byung-Yong Kim ${ }^{3}$, Jin-Kyu Rhee ${ }^{2}$, Jinjong Myoung ${ }^{4}$, Young-Do Nam ${ }^{5,6+}$ and Seong Woon Roh ${ }^{1,6^{*}+}$
}

\begin{abstract}
Background: Enterococcus faecalis, the type strain of the genus Enterococcus, is not only a commensal bacterium in the gastrointestinal tract in vertebrates and invertebrates, but also causes serious disease as an opportunistic pathogen. To date, genome sequences have been published for over four hundred E. faecalis strains; however, pathogenicity of these microbes remains complicated. To increase our knowledge of $E$. faecalis virulence factors, we isolated strain CBA7120 from the feces of an 81-year-old female from the Republic of Korea and performed a comparative genomic analysis.

Results: The genome sequence of E. faecalis CBA7120 is 3,134,087 bp in length, with a G + C content of 37.35 mol\%, and is comprised of four contigs with an N50 value of 2,922,046 bp. The genome showed high similarity with other strains of E. faecalis, including OG1RF, T13, 12107 and T20, based on OrthoANI values. Strain CBA7120 contains 374 pan-genome orthologous groups (POGs) as singletons, including "Phages, Prophages, Transposable elements, Plasmids," "Carbohydrates," "DNA metabolism," and "Virulence, Disease and Defense" subsystems. Genes related to multidrug resistance efflux pumps were annotated in the genome.
\end{abstract}

Conclusions: The comparative genomic analysis of E. faecalis strains presented in this study was performed using a variety of analysis methods and will facilitate future identification of hypothetical proteins.

Keywords: Enterococcus faecalis, Genome sequence, Comparative genomics, Virulence factors

\section{Background}

Genus Enterococcus is a common member of the normal intestinal flora in various species, both vertebrates and invertebrates. However, some species in the genus Enterococcus are leading causes of highly contagious hospital-acquired infections, including urinary tract, intra-abdominal, pelvic, and soft tissue infections, as well as bacteremia and endocarditis. Thus, members of the genus Enterococcus have been extensively studied. The genus Enterococcus was first described by Schleifer and Kilpper-Balz [1] and species of the genus Enterococcus

\footnotetext{
*Correspondence: seong18@gmail.com

${ }^{\dagger}$ Young-Do Nam and Seong Woon Roh contributed equally to this work

1 Biological Disaster Analysis Group, Korea Basic Science Institute,

Daejeon 34133, South Korea

Full list of author information is available at the end of the article
}

are Gram-positive, non-spore-forming, facultative anaerobic microbes that produce lactic acid. Enterococcus faecalis DSM $20478^{\mathrm{T}}$ is the type species for the genus [2].

The first genome sequence of Enterococcus was published by Paulsen in 2003 with E. faecalis V583 [2]. To date, more than 400 strains of E. faecalis have been sequenced and analyzed. The virulence factors present in E. faecalis are well established, and include aggregation substances, surface adhesins, sex pheromones, lipoteichoic acid, extracellular superoxide, the lytic enzymes gelatinase and hyaluronidase, and the toxin cytolysin, but novel virulence factors continue to be reported [3]. In this study, we performed sequencing and genomic analysis of E. faecalis CBA7120, isolated from the feces of an 81-year-old female. Comparison of genomic data from E. faecalis CBA7120 with other 
genomes of E. faecalis may improve our understanding of the virulence factors and pathogenesis present in Enterococcus.

\section{Methods}

\section{Strain isolation and DNA extraction}

Enterococcus faecalis CBA7120 was isolated from the feces of an 81-year-old healthy female living in the Republic of Korea and cultivated on modified EggerthGagnon (EG) medium [containing per liter of distilled water: peptone $10 \mathrm{~g}, \mathrm{Na}_{2} \mathrm{HPO}_{4} 4 \mathrm{~g}$, porcine gastric mucin $2 \mathrm{~g}$, sheep blood $50 \mathrm{ml}$, agar $15 \mathrm{~g}$ ] at $37^{\circ} \mathrm{C}$ for $24 \mathrm{~h}$ in an anaerobic chamber (Coy Laboratory Products). Once a pure culture was obtained, strain CBA7120 was preserved at $-80{ }^{\circ} \mathrm{C}$ in a suspension of $20 \%$ glycerol for long-term storage. Genomic DNA for sequencing was prepared using QuickGene DNA tissue kit S (Kurabo, Japan) and QIAamp DNA extraction Kits (Qiagen, USA).

\section{Whole genome sequencing, assembly, and gene annotation}

A SMRTbell library was constructed according to the Pacific Biosciences protocol "20-kb Template Preparation Using BluePippin Size-selection system (15-kb Size Cutoff)". The library was sequenced using P6-C4 chemistry on a Pacific Biosciences RS II instrument. The PacBio RS II sequencing system generated 150,292 reads, with an average read length of $8095 \mathrm{bp}$ from one SMRT cell. For the assembly, filtering was performed by Hierarchical Genome Assembly Process (HGAP) version 2 protocol with default parameter. Assembly was performed using the HGAP 2 protocol with default parameters in SMRT Analysis version 2.3 [4]. The assembly was polished with three successive passes through Quiver to reach a final consensus accuracy of $>99.988 \%$ at $232.798 \times$ coverage. Finally, finished assembly consisted of four contigs. Using RS_Modification_and_Motif_analysis protocol with default parameter in PacBio SMRT analysis pipeline, 4 N6-methyladenine and other six unidentified methylated motifs were identified. Gene prediction was accomplished using Glimmer3 [5] on the Rapid Annotation using Subsystem Technology (RAST; http://rast.nmpdr. org/) server [6], and gene annotation was performed using the SEED and Clusters of Orthologous Groups (COG; http://www.ncbi.nlm.nih.gov/COG/) databases [7, 8]. RNAmmer 1.2 [9] and tRNAscan-SE 1.21 [10] were used to identify rRNA and tRNA sequences, respectively.

\section{Multilocus sequence typing (MLST)}

MLST was performed using the following seven housekeeping genes: glucose-6-phosphate dehydrogenase ( $g d h)$, glyceraldehyde-3-phosphate dehydrogenase ( $g y d)$, phosphate ATP binding cassette transporter (pstS), glucokinase ( $g k i)$, shikimate-5-dehydrogenase (aroE), xanthine phosphoribosyltransferase $(x p t)$, and acetyl-CoA acetyltransferase (yiqL) using PubMLST (http://pubmlst. org/efaecalis/).

\section{Comparative genomic analysis}

Using data in the NCBI genome database (http://www. ncbi.nlm.nih.gov/genome/), four E. faecalis strains were selected as the closest neighbors of strain CBA7120 (>89\% symmetric identity): E. faecalis strain 12107, T13, OG1RF, and T20, and these genomes were used for comparative genomic analysis. The genome of E. faecalis TX0031 also showed high symmetric identity, but it was excluded from further analysis because the genome contains a large number of contigs. For whole-genome comparison, the genomes of strain CBA7120 and the other related strains were aligned using the progressive MAUVE algorithm in the MAUVE multiple genome alignment software 2.4.0 [11]. The OrthoANI algorithm was used to assess overall similarity between two genome sequences [12]. OrthoANI values were obtained and a phylogenetic tree was constructed based on OrthoANI analysis of the E. faecalis strains CBA7120, 12107, T13, OG1RF, and T20 using the orthologous average nucleotide identity tool [12]. Orthologs in the genomes of strain CBA7120 and other related strains were identified using reciprocal best hit (RBH) in the UBLAST program [13]. Pan-genome orthologous groups (POGs) were constructed using the EzBioCloud Comparative Genomics Database (http://cg.ezbiocloud.net/). For the visualization heat map and the dendrogram based on the gene content (presence or absence) of the genomes of strain CBA7120 and the other four strains, Jaccard coefficients and UPGMA clustering were used to calculate the presence of POGs and genome clustering, respectively. The Venn diagram based on POGs shared between strain CBA7120 and related strains was constructed using the jvenn program [14].

\section{Quality assurance}

To obtain pure cultures, a single colony of strain CBA7120 was repeatedly transferred to fresh modified 
EG medium more than three times, and confirmed using electron microscopy (Fig. 1). Genomic DNA of strain CBA7120 was purified using an MG Genomic DNA purification kit (Doctor Protein) and the 16S rRNA gene sequence from the draft genome was used to check for contamination.

\section{Results and discussion}

\section{General features}

General genome features of E. faecalis CBA7120 are shown in Table 1 . The genome sequence of strain CBA7120 is $3,134,087$ bp in length, with a $\mathrm{G}+\mathrm{C}$

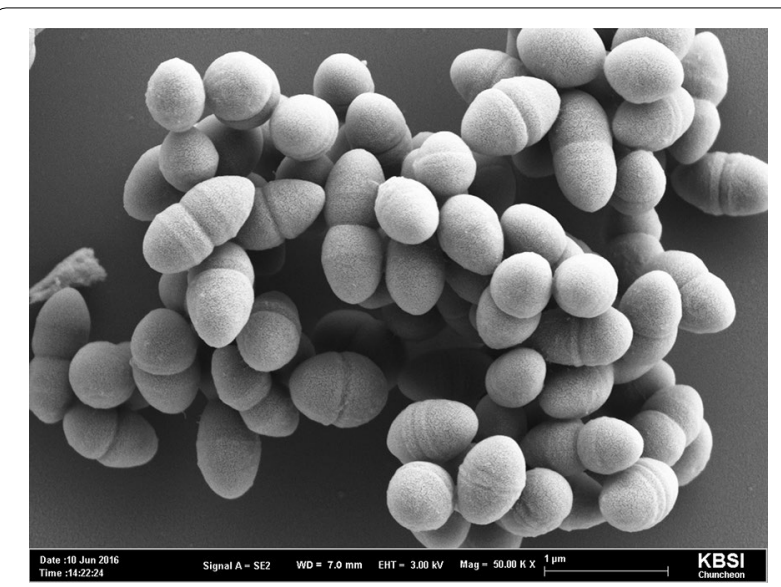

Fig. 1 Photomicrograph of Enterococcus faecalis CBA7120

Table 1 General genome features of Enterococcus faecalis CBA7120

\begin{tabular}{ll}
\hline Item & Values \\
\hline Finishing quality & Draft \\
Sequencing platforms & PacBio_20K \\
Assembler & PacBio SMRT Analysis 2.3.0 \\
Methods reads & 150,292 \\
Genome coverage & $232.798 X$ \\
Assembly size (bp) & $3,134,087$ \\
N50 & $2,922,046$ \\
DNA G + C content (\%) & 37.35 \\
Total contigs & 4 \\
Coding sequences & 3018 \\
rRNA genes & $4165-23 S-5 S$ rRNA operon \\
tRNA genes & 60 \\
\hline
\end{tabular}

content of $37.35 \mathrm{~mol} \%$, and is comprised of four contigs with an N50 value of 2,922,046 bp. Using BLAST in the NCBI, we found that contig 1,2 and 4 represented plasmid sequences and contig 3 represented chromosome sequence. The genome contains 3018 coding DNA sequences (CDSs), 60 tRNA genes, and four 16S-23S-5S rRNA operons. The distribution of COGs and SEED subsystems is illustrated in Fig. 2. The most abundant COG categories were G (carbohydrate transport and metabolism), $\mathrm{R}$ (general function prediction only), K (transcription), L (replication, recombination and repair), E (amino acid transport and metabolism), $J$ (translation, ribosomal structure and biogenesis), and $\mathrm{P}$ (inorganic ion transport and metabolism); the S category (function unknown) was also abundant (Fig. 2a). The SEED subsystems "Carbohydrates", "Amino Acids and Derivatives", "Protein Metabolism", "DNA Metabolism", "Cell Wall and Capsule", "Cofactors, Vitamins, Prosthetic Groups, Pigments", "RNA Metabolism”, and "Nucleosides and Nucleotides" subsystems were most abundant (Fig. 2b).

\section{Multilocus sequence typing (MLST)}

Enterococcus faecalis CBA7120 was typed with MLST at loci gdh 1, gyd 7, pstS 9, gki 1, aroE 1, xpt 3, and yqiL 1, and was classified as sequence type 5 (ST 5 ) containing solely strain B343 that was isolated from a chicken product in Spain.

\section{Comparative genomics of strain CBA7120 with other $E$. faecalis strains}

Whole-genome comparison of strain CBA7120 with $E$. faecalis 12107, T13, OG1RF, and T20 showed that most of the locally collinear blocks (LCBs) are highly homologous between the five assemblies, although the genome of strain CBA7120 contains large gaps in three LCBs (Fig. 3). E. faecalis CBA7120 showed 99.24, 99.55, 99.62 and $99.64 \%$ orthoANI values with $E$. faecalis strains OG1RF, T13, 12107, and T20, respectively. The phylogenetic tree based on orthoANI values for strain CBA7120 and the four reference strains indicated that strain CBA7120 is closely related to the E. faecalis reference strains (Fig. 4a). Based on analysis of the gene presence or absence heat map, strain CBA7120 possesses different POGs from the other four strains. In the dendrogram based on presence of POGs, strain CBA7120 was located as an outgroup to the other E. faecalis strains (Fig. 4b). As shown in Fig. 5, strain CBA7120 and the 
a

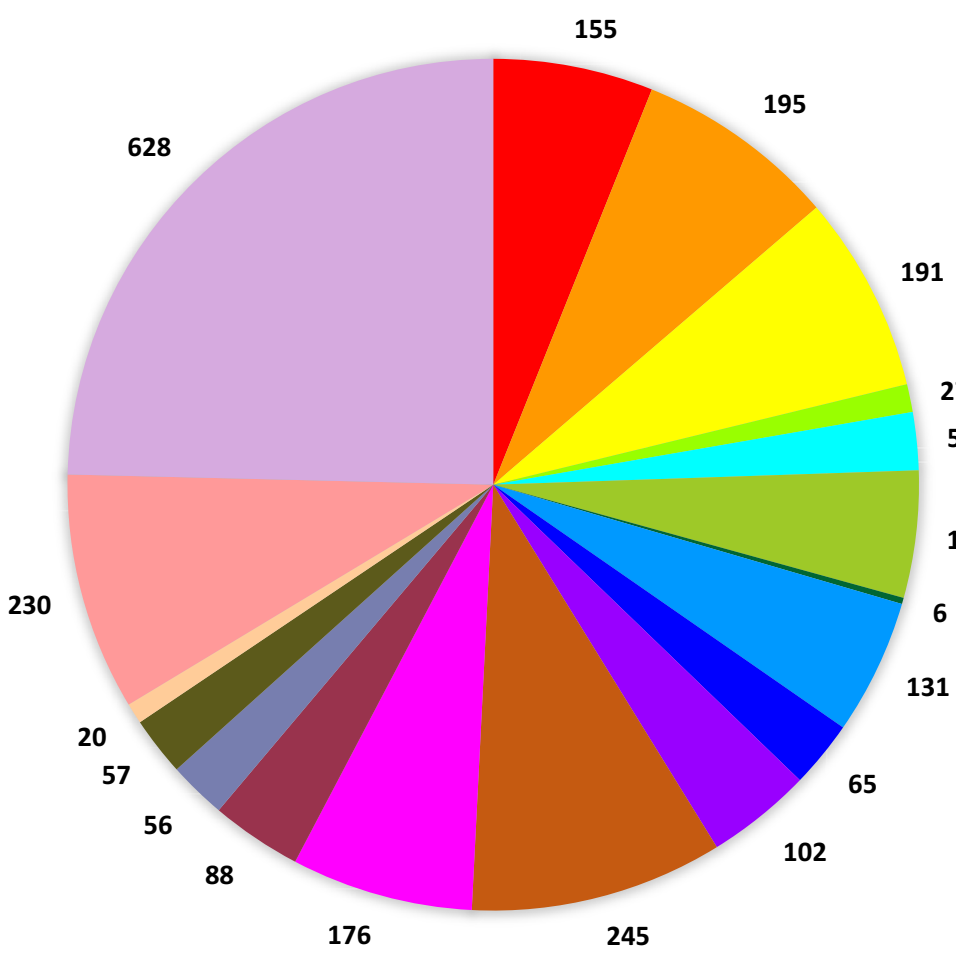

b

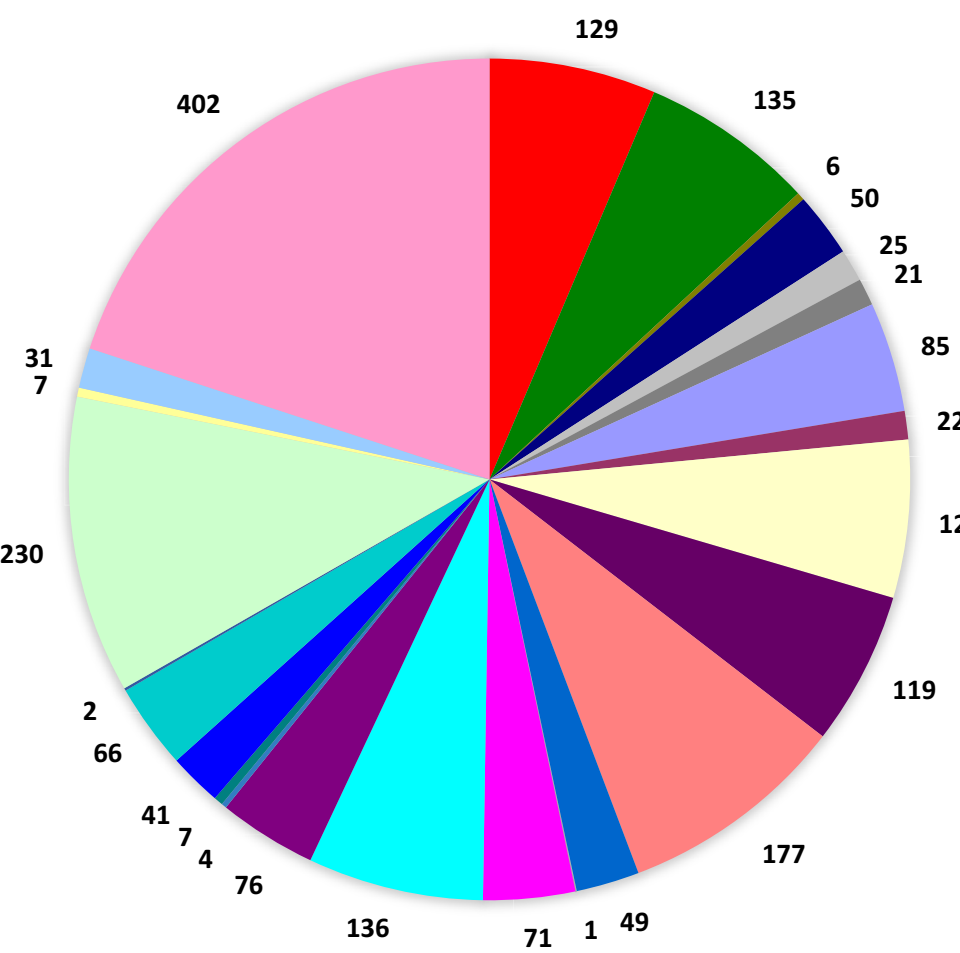

- Translation, ribosomal structure and biogenesis (155)

- Transcription (195)

Replication, recombination and repair (191)

-Cell cycle control, cell division, chromosome partitioning (27)

-Posttranslational modification, protein turnover, chaperones (56)

27

Cell wall/membrane/envelope biogenesis (123)

Cell motility (6)

- Inorganic ion transport and metabolism (131)

- Signal transduction mechanisms (65)

123

- Energy production and conversion (102)

- Carbohydrate transport and metabolism (245)

- Amino acid transport and metabolism (176)

- Nucleotide transport and metabolism (88)

- Coenzyme transport and metabolism (56)

- Lipid transport and metabolism (57)

- Secondary metabolites biosynthesis, transport and catabolism (20)

-General function prediction only (230)

Function unknown (628)

- Cofactors, Vitamins, Prosthetic Groups, Pigments (129)

- Cell Wall and Capsule (135)

- Potassium metabolism (6)

- Virulence, Disease and Defense (50)

Photosynthesis (0)

Miscellaneous (25)

- Phages, Prophages, Transposable elements, Plasmids (21)

Membrane Transport (85)

- Iron acquisition and metabolism (22)

RNA Metabolism (122)

- Nucleosides and Nucleotides (119)

Protein Metabolism (177)

- Cell Division and Cell Cycle (49)

Motility and Chemotaxis (1)

Regulation and Cell signaling (71)

$\checkmark$ Secondary Metabolism (0)

DNA Metabolism (136)

Fatty Acids, Lipids, and Isoprenoids (76)

- Nitrogen Metabolism (4)

Dormancy and Sporulation (7)

- Respiration (41)

- Stress Response (66)

Metabolism of Aromatic Compounds (2)

Amino Acids and Derivatives (230)

Sulfur Metabolism (7)

Phosphorus Metabolism (31)

Carbohydrates (402)

Fig. 2 Analysis of annotated genes for Enterococcus faecalis CBA7120 based on the SEED (a) and COG (b) databases 


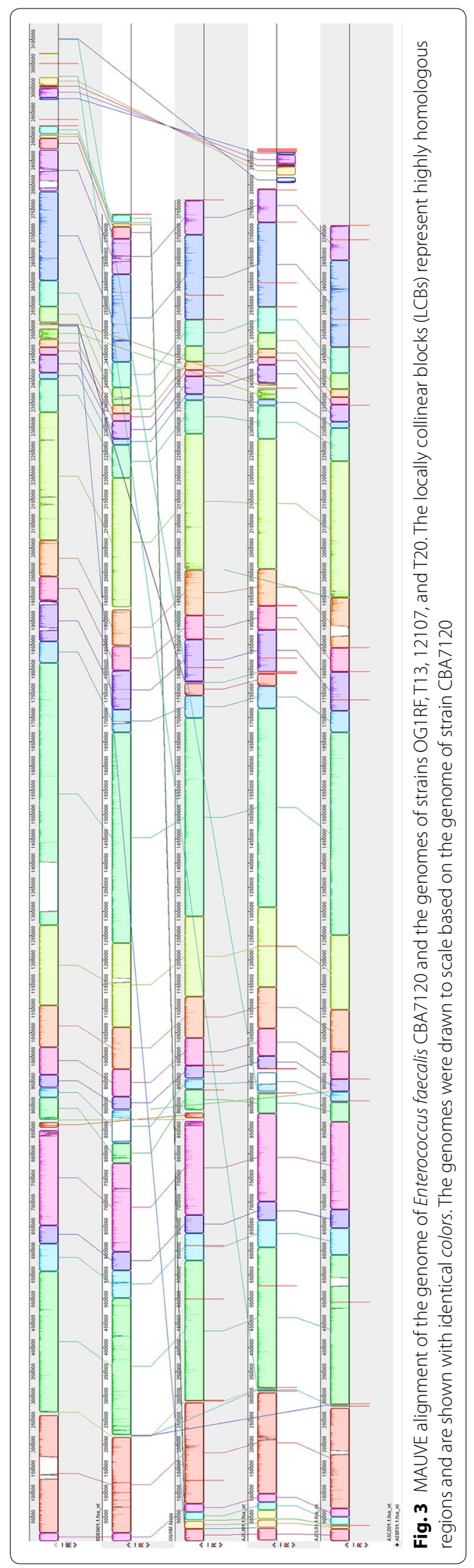


a

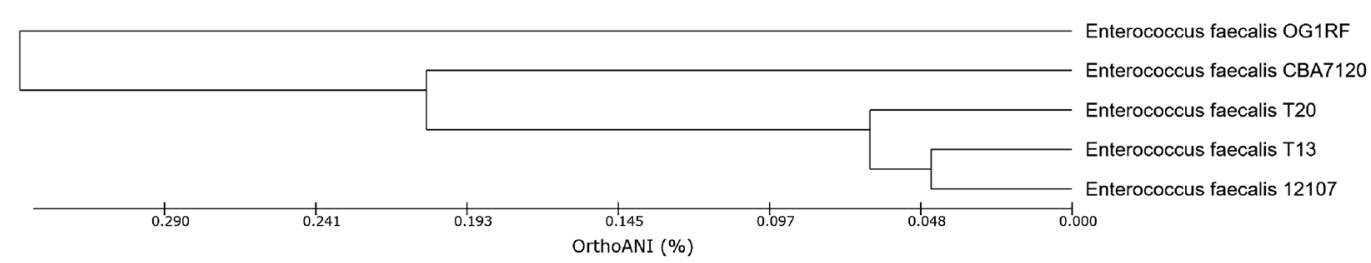

$\mathbf{b}$
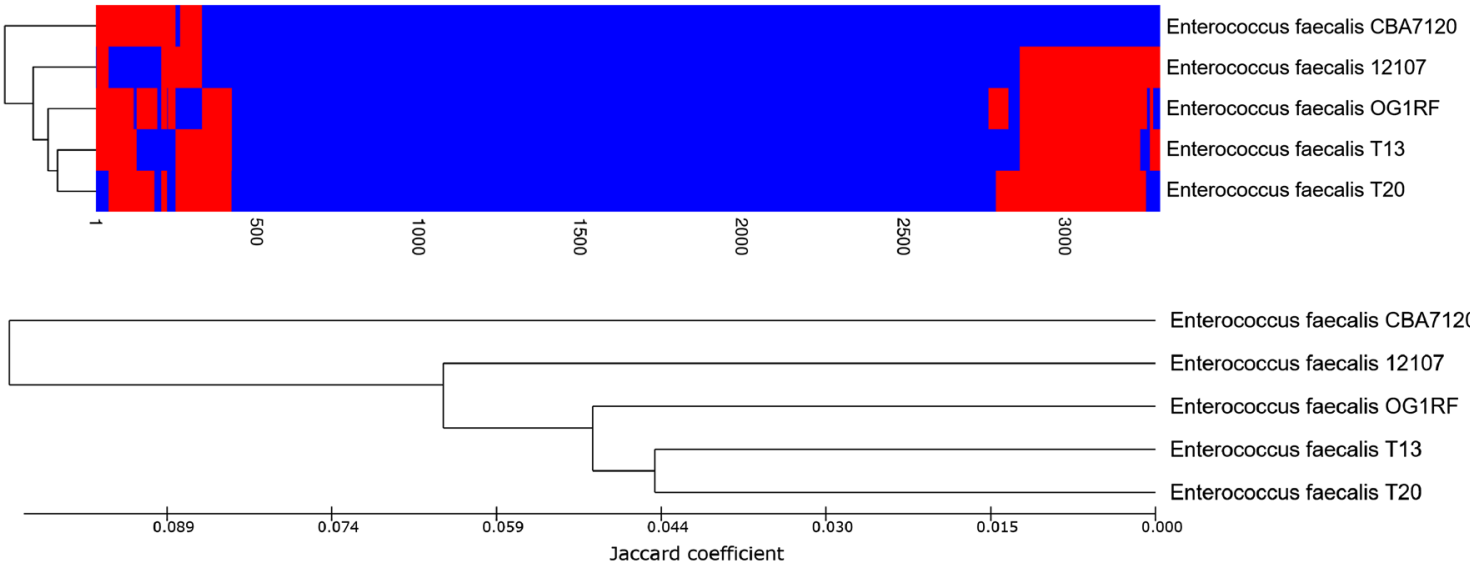

Fig. 4 Dendrogram based on OrthoANI analysis and the presence of POGs in the genome of Enterococcus faecalis CBA7120 and the genomes of strains OG1RF, T13, 12107, and T20. a ANI phylogenetic tree. Using the orthologous average nucleotide identity tool, the phylogenetic tree was constructed based on OrthoANI values. b Dendrogram based on presence of POGs. Using Jaccard coefficients and UPGMA clustering, a dendrogram was generated. Blue indicates present genes and red indicates absent genes

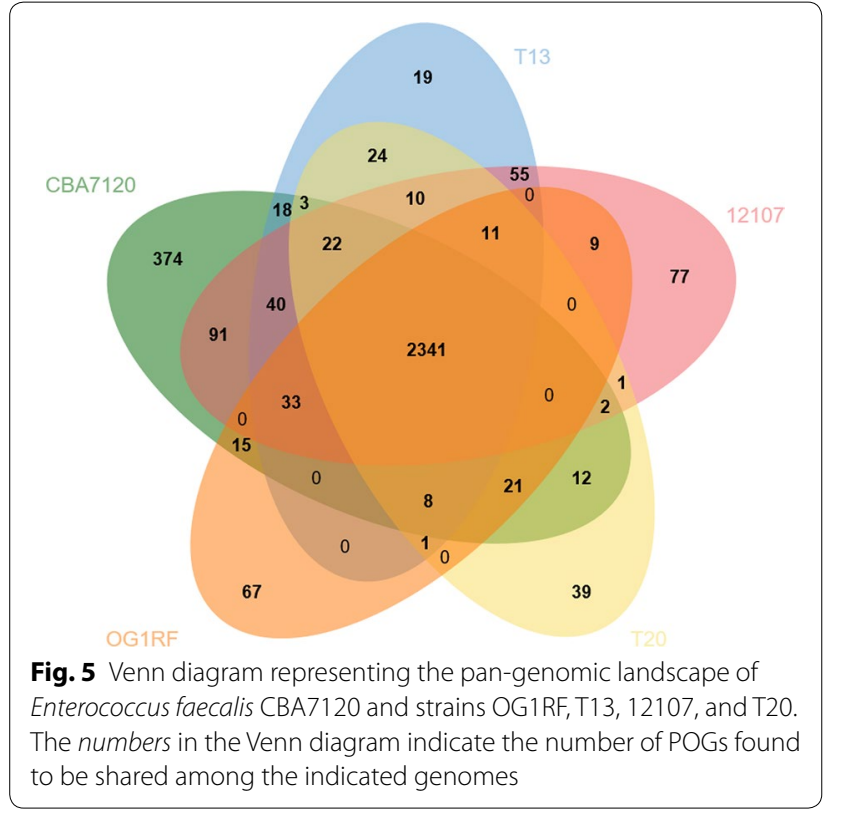

other four strains share 2341 POGs. The genome of strain CBA7120 contains only 374 POGs as singletons, contained in a single genome. Without the POGs that had no match in the SEED database, most of the 91 POGs identified belong to the "Phages, Prophages, Transposable elements, Plasmids" (20 POGs), "Carbohydrates" (12 POGs), "DNA metabolism" (11 POGs), and "Virulence, Disease and Defense" subsystems (9 POGs).

\section{Virulence factors}

Based on comparison with the SEED database, 28 CDS were annotated as belonging to the "Virulence, Disease, Defense" category (Table 2). Strain CBA7120 was found to contain genes related to multidrug resistance efflux pumps, such as multidrug resistance efflux pump PmrA, multi antimicrobial extrusion protein, multidrug and toxin extrusion (MATE) family efflux pump YdhE/NorM, and multidrug-efflux transporter. 
Table 2 Summary of protein coding sequences annotated as belonging to the "Virulence, Disease, and Defense" subsystem in the SEED database

\begin{tabular}{|c|c|c|}
\hline Subsystem & Role & Location \\
\hline Streptococcus pyogenes recombinatorial zone & Chaperonin (heat shock protein 33) & Contig 3 \\
\hline \multirow[t]{6}{*}{ Colicin V and bacteriocin production cluster } & Dihydrofolate synthase & Contig 3 \\
\hline & Folylpolyglutamate synthase & Contig 3 \\
\hline & Amidophosphoribosyltransferase & Contig 3 \\
\hline & Acetyl-coenzyme A carboxyl transferase beta chain & Contig 3 \\
\hline & Colicin V production protein & Contig 3 \\
\hline & tRNA pseudouridine synthase $A$ & Contig 3 \\
\hline \multirow[t]{3}{*}{ Copper homeostasis } & Negative transcriptional regulator-copper transport operon & Contig 3 \\
\hline & Copper-translocating P-type ATPase & Contig 3 \\
\hline & Copper chaperone & Contig 3 \\
\hline \multirow[t]{3}{*}{ Cobalt-zinc-cadmium resistance } & DNA-binding heavy metal response regulator & Contig 3 \\
\hline & Cobalt-zinc-cadmium resistance protein & Contig 3 \\
\hline & Transcriptional regulator, MerR family & Contig 3 \\
\hline \multirow[t]{4}{*}{ Resistance to fluoroquinolones } & DNA gyrase subunit $B$ & Contig 3 \\
\hline & DNA gyrase subunit A & Contig 3 \\
\hline & Topoisomerase IV subunit B & Contig 3 \\
\hline & Topoisomerase IV subunit A & Contig 3 \\
\hline \multirow[t]{2}{*}{ Copper homeostasis: copper tolerance } & Cytoplasmic copper homeostasis protein CutC & Contig 3 \\
\hline & Magnesium and cobalt efflux protein CorC & Contig 3 \\
\hline \multirow[t]{3}{*}{ Beta-lactamase } & Beta-lactamase class $C$ and other penicillin-binding proteins & Contig 3 \\
\hline & Beta-lactamase & Contig 3 \\
\hline & Metal-dependent hydrolases of the beta-lactamase superfamily I & Contig 3 \\
\hline \multirow[t]{2}{*}{ Cadmium resistance } & Cadmium resistance protein & Contig 1 \\
\hline & Cadmium efflux system accessory protein & Contig 1 \\
\hline \multirow[t]{4}{*}{ Multidrug resistance efflux pumps } & Multidrug resistance efflux pump PmrA & Contig 3 \\
\hline & $\begin{array}{l}\text { Multi antimicrobial extrusion protein }[\mathrm{Na}(+) / \text { drug antiporter], MATE family of MDR efflux } \\
\text { pumps }\end{array}$ & Contig 3 \\
\hline & Multidrug and toxin extrusion (MATE) family efflux pump YdhE/NorM, homolog & Contig 3 \\
\hline & Multidrug-efflux transporter, major facilitator superfamily (MFS) & Contig 3 \\
\hline
\end{tabular}

\section{Future directions}

Genomic analysis based on ortholog analysis may be a powerful comparative genomics tool. Additional study of the virulence factors present in E. faecalis CBA7120 will guide further research on $E$. faecalis virulence.

\begin{abstract}
Abbreviations
CDSs: coding DNA sequences; COG: clusters of orthologous groups; EG: Eggerth-Gagnon medium; LCB: locally collinear block; MATE: multidrug and toxin extrusion; MLST: multilocus sequence typing; POG: pan-genome orthologous group; RAST: rapid annotation using subsystem technology; RBH: reciprocal best hit.
\end{abstract}

\section{Authors' contributions}

YDN and SWR designed and coordinated all the experiments. HSS characterized the strain and prepared the high-quality DNA. JYK, YJC, BYK performed the sequence assembly, gene prediction, gene annotation, comparative genomic analysis and wrote manuscript. YBK, JK, JSC, JKR and JM checked and edited the manuscript. All authors read and approved the final manuscript.

\begin{abstract}
Author details
${ }^{1}$ Biological Disaster Analysis Group, Korea Basic Science Institute, Daejeon 34133, South Korea. ${ }^{2}$ Department of Food Science and Engineering, Ewha Womans University, Seoul 03760, South Korea. ${ }^{3}$ ChunLab Inc., Seoul National University, Seoul 151-742, South Korea. ${ }^{4}$ Korea Zoonosis Research Institute, Chonbuk National University, Jeonju 561-756, South Korea. ${ }^{5}$ Research Group of Gut Microbiome, Korea Food Research Institute, Seongnam 13539, South Korea. ${ }^{6}$ University of Science and Technology, Daejeon 34113, South Korea.
\end{abstract}

\section{Acknowledgements}

Not applicable.

\section{Competing interests}

The authors declare that they have no competing interests. 


\section{Availability of data and materials}

This Whole Genome Shotgun project has been deposited at DDBJ under the accession numbers BDEN01000001-BDEN01000004.

\section{Ethics approval and consent to participate}

This study was approved by the Institutional Review Board of the Korea National Institutes of Health $(\mathrm{NIH})$ under approval number 2015-02-EXP-05-3C-A.

\section{Funding}

This research was supported from the Basic Science Research Program through the National Research Foundation of Korea (NRF) funded by the Ministry of Education, Science, and Technology (2015R1D1A1A09061039), from the Center for Analytical Research of Disaster Science of the Korea Basic Science Institute (C36703), from Korean Food Research Institute (E0131600-04) and from Korea Centers for Disease Control and Prevention (4847-311-210-13, 2015).

Received: 24 August 2016 Accepted: 19 November 2016

Published online: 25 November 2016

\section{References}

1. Schleifer KH, Kilpper-Bälz R. Transfer of Streptococcus faecalis and Streptococcus faecium to the Genus Enterococcus nom. rev. as Enterococcus faecalis comb. nov. and Enterococcus faecium comb. nov. Int J Syst Evol Microbiol. 1984;34:31-4

2. Paulsen IT, Banerjei L, Myers GS, Nelson KE, Seshadri R, Read TD, et al. Role of mobile DNA in the evolution of vancomycin-resistant Enterococcus faecalis. Science. 2003:299:2071-4.

3. Kayaoglu G, Orstavik D. Virulence factors of Enterococcus faecalis: relationship to endodontic disease. Crit Rev Oral Biol Med. 2004;15:308-20.
4. Chin CS, Alexander DH, Marks P, Klammer AA, Drake J, Heiner C, et al. Nonhybrid, finished microbial genome assemblies from long-read SMRT sequencing data. Nat Methods. 2013;10:563-9.

5. Delcher AL, Bratke KA, Powers EC, Salzberg SL. Identifying bacterial genes and endosymbiont DNA with Glimmer. Bioinformatics. 2007;23:673-9.

6. Aziz RK, Bartels D, Best AA, DeJongh M, Disz T, Edwards RA, et al. The RAST server: rapid annotations using subsystems technology. BMC Genomics. 2008;9:75.

7. Galperin MY, Makarova KS, Wolf YI, Koonin EV. Expanded microbial genome coverage and improved protein family annotation in the COG database. Nucleic Acids Res. 2015;43:D261-9.

8. Overbeek R, Begley T, Butler RM, Choudhuri JV, Chuang HY, Cohoon M, et al. The subsystems approach to genome annotation and its use in the project to annotate 1000 genomes. Nucleic Acids Res. 2005;33:5691-702.

9. Lagesen K, Hallin P, Rodland EA, Staerfeldt HH, Rognes T, Ussery DW. RNAmmer: consistent and rapid annotation of ribosomal RNA genes. Nucleic Acids Res. 2007:35:3100-8.

10. Lowe TM, Eddy SR. tRNAscan-SE: a program for improved detection of transfer RNA genes in genomic sequence. Nucleic Acids Res. 1997;25:955-64.

11. Darling AC, Mau B, Blattner FR, Perna NT. Mauve: multiple alignment of conserved genomic sequence with rearrangements. Genome Res. 2004;14:1394-403.

12. Lee I, Kim YO, Park SC, Chun J. OrthoANI: an improved algorithm and software for calculating average nucleotide identity. Int J Syst Evol Microbiol. 2016;66:1100-3.

13. Ward N, Moreno-Hagelsieb G. Quickly finding orthologs as reciprocal best hits with BLAT, LAST, and UBLAST: how much do we miss? PLOS ONE. 2014:9:e101850.

14. Bardou P, Mariette J, Escudie F, Djemiel C, Klopp C. jvenn: an interactive Venn diagram viewer. BMC Bioinformatics. 2014;15:293.

\section{Submit your next manuscript to BioMed Central and we will help you at every step:}

- We accept pre-submission inquiries

- Our selector tool helps you to find the most relevant journal

- We provide round the clock customer support

- Convenient online submission

- Thorough peer review

- Inclusion in PubMed and all major indexing services

- Maximum visibility for your research

Submit your manuscript at www.biomedcentral.com/submit 